\title{
Disruption of Sequential Priming in Organic and Pharmacological Amnesia: A Role for the Medial Temporal Lobes in Implicit Contextual Learning
}

\author{
David R Shanks*,', Shelley Channon', Leonora Wilkinson ${ }^{2}$ and H Valerie Curran' \\ 'Department of Psychology, University College London, London, UK; ${ }^{2}$ Institute of Neurology, University College London, London, UK
}

\begin{abstract}
We examined learning and expression of contextual implicit learning of a sequence of targets in a speeded target-detection task in amnesic and control participants. Amnesia was of organic origin in one participant group and induced psychopharmacologically (diazepam 7.5 or $15 \mathrm{mg}$ ) in another. Although the amnesic groups were able to learn the target sequence normally, their expression of sequence knowledge (priming) was attenuated when contextual support was limited. This was evaluated by studying response latencies for targets primed by between 0 and 5 preceding context locations. Whereas control participants showed priming when the current target location was primed by only two previous locations, priming was eliminated with two (but not four) previous locations by a low dose of diazepam and was eliminated even with four elements of context under a high dose of diazepam and in amnesia of organic origin. The results suggest that a function of the hippocampal memory system is to support contextual learning and performance, even when that learning is nondeclarative.

Neuropsychopharmacology (2006) 31, I768- 1776. doi:I0.1038/sj.npp. 1300935; published online 12 October 2005
\end{abstract}

Keywords: learning; amnesia; diazepam; benzodiazepine; memory; priming

\section{INTRODUCTION}

One view of the memory function of the hippocampus and medial temporal lobes is that they control explicit or declarative memory (Gabrieli, 1998; Knowlton, 2005; Squire, 1994), referring to the conscious recollection of prior events or episodes. An alternative view proposes that these structures are particularly involved in binding events to the contexts in which they occur, with explicit/declarative memory being a special case of such contextual or relational memory (Cohen and Eichenbaum, 1993; Cohen et al, 1999; O'Reilly and Rudy, 2001; Rudy and Sutherland, 1995). Consider learning a list of words in a certain context such as a particular experimental room. The declarative view proposes that this form of learning is only hippocampal dependent to the extent that the memory is retrieved via conscious recollection; if it is accessed implicitly (eg via a priming test) then the hippocampus plays no role. In contrast, the relational theory proposes that binding

\footnotetext{
* Correspondence: Professor DR Shanks, Department of Psychology, University College London, Gower Street, London WCIE 6BY, UK Tel: + 44207679 7588, Fax: + 44207436 4276,

E-mail: d.shanks@ucl.ac.uk

Received 6 May 2005; revised I August 2005; accepted 8 September 2005

Online publication: 13 September 2005 at http://www.acnp.org/ citations/Npp09| 305050304/default.pdf
}

together the word list with the context intrinsically requires the hippocampus, regardless of how memory is probed. On this theory, learning about noncontextual aspects of the list (eg single-item learning) can proceed normally without the hippocampus, but not learning which requires items to be bound together in memory.

A key prediction which distinguishes these accounts is that the contextual memory theory, but not the declarative theory, proposes that the medial temporal lobe memory system plays a role in contextual memory even when that memory is probed implicitly or indirectly via a priming measurement (Chun and Phelps, 1999). Here we provide support for the contextual memory theory by demonstrating that the expression of perceptual-motor sequence knowledge in a priming procedure is attenuated in both organic and pharmacological amnesia. We show that the extent of dysfunction in these structures correlates with the amount of context needed to induce priming.

Participants initially learned a sequence of target movements in a speeded choice reaction time (RT) task. On each trial a target appeared at one of four horizontally aligned locations on a display and the participant responded with the appropriate response key as quickly as possible. The next target then appeared immediately and the participant again made a localization response. Rather than moving at random, the target followed a noisy repeating sequence in which on each trial its location was predictable with 
probability 0.85 ; on other trials $(p=0.15)$ it appeared at an improbable location. Across a total of 900 (Study 1) or 1200 (Study 2) training trials participants therefore had the opportunity to learn the probabilistic sequential structure determining target location (Cleeremans and McClelland, 1991; Schvaneveldt and Gomez, 1998; Shanks et al, 2003). The precise nature of implicit learning, and what characterizes a learning episode as implicit, is controversial (Cleeremans et al, 1998; Shanks, 2005; Shanks and St. John, 1994); However, the serial RT task used in the present study is implicit in the sense that learning occurs in the absence of intention to learn (Cleeremans and Jiménez, 1998), can proceed near normally in amnesia (Reber and Squire, 1998), and the acquired knowledge is difficult to verbalize or recognize (Chun and Phelps, 1999; Willingham et al, 1989).

In the critical test phase participants were presented with sequences of six targets to which they again made speeded localization responses. Half of the sequences were familiar in that they comprised entirely predictable targets, and half were unfamiliar in comprising a series of unpredictable movements. We have demonstrated priming in these test trials in that familiar sequences are executed faster than new ones (Shanks and Perruchet, 2002; Shanks et al, 2003): as if they are attaining a state of response 'flow,' participants are able to use each stimulus and response as a cue for the subsequent one. Crucially, however, this test method allows us to determine how many preceding targets are needed to induce priming. In normal participants, priming requires two preceding targets with the present methods (Shanks et al, 2003).

The role of the medial temporal lobe memory system was explored by studying both a group of patients with confirmed organic amnesia (compared to a matched control group) and in three groups of participants who were administered one of two doses of the amnestic drug diazepam or a placebo.

Benzodiazepines (BZDs) like diazepam act via specific BZD receptors to facilitate the transmission of GABA $(\gamma-$ aminobutyric acid), the brain's major inhibitory neurotransmitter. BZD receptors are widely distributed in the brain but are most concentrated in the limbic system (including the hippocampus) and the cerebral cortex. Diazepam induces a robust and dose-dependent anterograde impairment on explicit measures of memory while leaving performance on implicit measures such as perceptual and conceptual priming intact (see Curran, 2000, for a review). We anticipated here that our implicit measure of procedural learning would be similarly unaffected by diazepam because procedural learning is preserved following the BZDs lorazepam and oxazepam (Bishop et al, 1996; Knopman, 1991; Martin et al, 2002; Nissen et al, 1987).

\section{METHODS}

The experiment was approved by the ethics committee of University College London. In all, 10 participants (six males and four females) with memory disorders took part in Study 1. Four of these were diagnosed with alcoholic Korsakoff's syndrome. Four had developed memory problems from suspected temporal lobe damage following encephalitis, and MRI radiological investigation available in two of these cases confirmed medial temporal lobe involvement. Two had unilateral medial temporal lobe lesions, one of whom had a left-sided posterior communicating artery infarct and the other a right-sided tumor, both confirmed by radiological investigation. In all, 22 control participants also took part in the study (14 males and eight females). To be included in the study, participants had to be between 18 and 75 years of age, fluent in English, within the normal range on the Test of Reception of Grammar (Bishop, 1989), and have an IQ score of 85 or above. Exclusion criteria included significant physical or psychiatric illness (with the exception of the primary diagnosis for the amnesic group), hydrocephalus and dementing conditions, and expressive or receptive aphasia. The two groups of participants did not differ significantly $(p>0.1)$ in age, years of education, National Adult Reading Test (Nelson, 1991) IQ, WAIS-R IQ (Wechsler, 1981), forward digit span, forward spatial span, or perceptual performance on the VOSP (Warrington and James, 1991) incomplete letters or position discrimination. Participants were paid for completing the study.

All participants in Study 1 were assessed on standardized clinical memory measures in addition to the experimental task. Measures of recall and recognition memory consisted of the Story Recall test from the Adult Memory and Information Processing Battery (Coughlan and Hollows, 1985); memory for a geometric drawing, the Rey-Osterreith figure (Rey, 1941); and memory for words and faces from the Recognition Memory Test (Warrington, 1984). The amnesic group performed significantly more poorly than the control group on each of these measures $(p<0.01)$ : Story Recall delayed, control: $M=72.6 \%$, amnesic: $M=$ 29.5\%; Rey-Osterreith figure delayed recall, control: $M=$ 64.3\%; amnesic: $M=28.2 \%$; RMT for words, control: $M=97.3 \%$, amnesic: $M=79.4 \%$; RMT for faces, control: $M=90.5 \%$, amnesic: $M=76.4 \%$. Degree of memory impairment in individual amnesic participants ranged from mild to marked; as expected, those with Korsakoff's syndrome tended to show the most severe impairment. The groups did not differ significantly $(p>0.05)$ on the Wisconsin Card Sorting test (Grant and Berg, 1948) number of correct sorting categories or number of perseverative errors but they did perform significantly more poorly $(p<0.05)$ than the control group on the Six Elements test (Shallice and Burgess, 1991) profile score and on the Chicago fluency test (Thurstone and Thurstone, 1962) number of ' $S$ ' words generated in $5 \mathrm{~min}$.

In Study 2, 48 healthy volunteers (24 male, 24 female) were recruited who fitted the relevant medical inclusion/ exclusion criteria. They were aged between 19 and 51 years $($ mean $=24)$. Males ranged in weight from 55 to $100 \mathrm{~kg}$ $($ mean $=74.00 \mathrm{~kg})$ and females from 49 to $76 \mathrm{~kg}$ (mean $=$ $59.59 \mathrm{~kg}$ ). Participants were instructed to abstain from alcohol for at least $24 \mathrm{~h}$ prior to the commencement of the study. If taking part in the morning they were asked to avoid or have a light low-fat breakfast and if taking part in the afternoon, to abstain from or to have a light low-fat lunch. The study was carried out in accordance with the Declaration of Helsinki. It was established that participants were not currently taking any medication at the time of test and were unfamiliar with the experimental task. Participants were paid a turn up fee of $£ 12$ plus an extra amount calculated according to their recognition performance as detailed below. 
Participants were randomly assigned to three groups (eight females and eight males per group): a placebo group, a diazepam $7.5 \mathrm{mg}$ (low dose) group, and a $15 \mathrm{mg}$ (high dose) group. Matched opaque gelatine capsules containing $7.5 \mathrm{mg}$ diazepam, $15 \mathrm{mg}$ diazepam, or lactose placebo were given orally. To investigate the effect of diazepam on sequence learning during its peak plasma concentration a delay of $40 \mathrm{~min}$ followed administration of the capsules before the main test session commenced.

Before and after treatment adminstration, participants rated their subjective mood on a 16-item visual analogue mood rating scale (Bond and Lader, 1974). They were also given prose recall tasks (Rivermead Behavioural Memory Test (Wilson et al, 1985), immediate and delayed) to assess the effects of diazepam on explicit memory. Results indicated that in the placebo group pre- and postadministration immediate and delayed recall scores were not different. For the low-dose group, the delayed $(p<0.05$, one-tailed) but not immediate recall score was lowered by the drug. For the high-dose group both immediate $(p<0.01)$ and delayed $(p<0.001)$ recall was impaired.

\section{Sequential RT Task}

The sequential RT (SRT) task was fully automated. Stimulus presentation, RT measurement, and response recording were all implemented on desktop computers with color monitors. Four boxes were arranged horizontally along the bottom of the computer screen in white against a blue background. The boxes were $38 \mathrm{~mm}$ wide and $23 \mathrm{~mm}$ high. On each trial, a white dot ( $2 \mathrm{~mm}$ diameter) appeared in the center of one of the boxes. The boxes will henceforth be referred to as locations A-D from left to right.

Two second-order conditional sequences $(S O C 1=C-A-D-$ C-B-D-B-A-C-D-A-B, SOC2 = D-C-A-B-D-A-C-B-A-D-B-C) were used in the target location task. These sequences are equated with respect to location frequency (each location occurs three times), first-order transition frequency (each location is preceded once by each of the other three locations), and repetitions (no repetitions in either sequence) (Reed and Johnson, 1994). The only difference between the sequences is in their second- and higher-order conditional structure. For counterbalancing purposes, about half the participants in each group of Study 1 (12 in the control group and seven in the amnesic group) trained on the SOC1 sequence and the remainder on the SOC2 sequence. In Study 2, half the participants in each condition trained on the SOC1 sequence and the remainder on the SOC2 sequence. Thus eight males and eight females in each group were trained on SOC1 $(n=4)$, and SOC2 $(n=4)$ respectively. One participant in the low-dose group, trained on SOC2 was excluded from the SRT analysis on the basis that her RTs were found to be exceptionally slow (three SDs above the mean). Hence for the low-dose group $n=15$ (eight males and seven females).

During the training phase (Figure 1a) the location of the target was specified by the assigned training sequence with probability 0.85 and by the alternate sequence with probability 0.15 . The probabilistic sequences were implemented by using the two most recent events to select the next event. With probability 0.85 , the next target would be the event in the training sequence specified by the last two locations and with probability 0.15 it would be the event in the alternate sequence specified by the last two locations. For example, if SOC1 was the training sequence for a given participant then the transition D-A was followed by a target at location B (following the specified sequence of SOC1) with a probability of 0.85 and it was followed by a target at location C (following the specified sequence of SOC2) with a probability of 0.15 . This algorithm was applied on each trial and determined the location of the current target simply on the basis of the two preceding targets. Improbable trials were equally distributed across the 12 elements of the sequence.

Although any given target was predicted (at $p=0.85$ ) by its two predecessors, it is important to emphasize that longer-range predictive relationships are also present in the stimuli. This can be understood by analogy to the alphabet. A given letter is perfectly predicted by its predecessor $(Q$ predicts $\mathrm{R}$ ) but at the same time it is also predicted by earlier letters (the 4th letter after $\mathrm{N}$ is $\mathrm{R}$ ). Thus, targets $n-3$ and $n-2$ predict the target on trial $n$ with probability $p=0.72\left(=0.85^{2}\right)$, targets $n-4$ and $n-3$ predict it with probability $p=0.61\left(=0.85^{3}\right)$, and so on. Each target is perfectly determined by any earlier pair of letters but for the possible intrusion of improbable trials, so each preceding pair of targets predicts the target on trial $n$ with slightly less certainty. Indeed, compared to the predictability of a target in a random sequence $(p=0.25)$, there is still an appreciable degree of predictability $(p=0.38)$ of a target at trial $n$ given information about targets as far back as trials $n-7$ and $n-6$. Thus, an efficient learning system will be able to exploit both long- and short-range contingencies in this task and benefit from them in much the same way as happens in statistical language learning (Elman, 1990). This is important for the contextual manipulation employed in the test phase.

The learning phase comprised nine (Study 1) or 12 (Study 2) 100-trial training blocks during which participants were exposed to a four-choice serial RT task. On each trial, participants reacted to the location of a dot as quickly as possible by pressing the corresponding key. Keys V, B, $\mathrm{N}$, and $\mathrm{M}$ corresponded to locations $\mathrm{A}-\mathrm{D}$, respectively. Participants were required to respond to locations $A$ and $B$ with the middle and index fingers, respectively, of their left hand and to locations $C$ and D with the index and middle finger, respectively, of their right hand. Participants were told to respond to the target as fast and as accurately as possible.

Each block of target-location trials began at a random point in the sequence. A target location trial ended when a participant pressed the correct key, at which time the target was erased. Response latencies were measured from the onset of the target to the completion of a correct response and errors were recorded. The next target appeared after a response-stimulus interval (RSI) of $0 \mathrm{~ms}$. This RSI was employed because some previous work has suggested that it is optimal for eliciting implicit learning (Destrebecqz and Cleeremans, 2001). RTs for participants trained with SOC1 and SOC2 as their probable sequence were combined in the following analyses. Trials on which an error was made were not included in the calculation of mean RT. RTs to the first two targets of each block were excluded from the analysis since their locations cannot be predicted. 
a Training stage:

base training sequence: CADCBDBACDAB

sample 100-trial training sequence with added noise:

$\mathrm{BACD} \underline{\boldsymbol{C}} \mathrm{BDBACDABCADCBDBACD} \underline{\boldsymbol{C}} \mathrm{BDB} \underline{\boldsymbol{C}}$ A $\underline{\boldsymbol{B}} \mathrm{CADCBDBA} \underline{\boldsymbol{D}} \mathrm{CBD} \underline{\boldsymbol{A}} \mathrm{BCADCBD}$

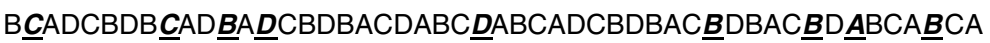

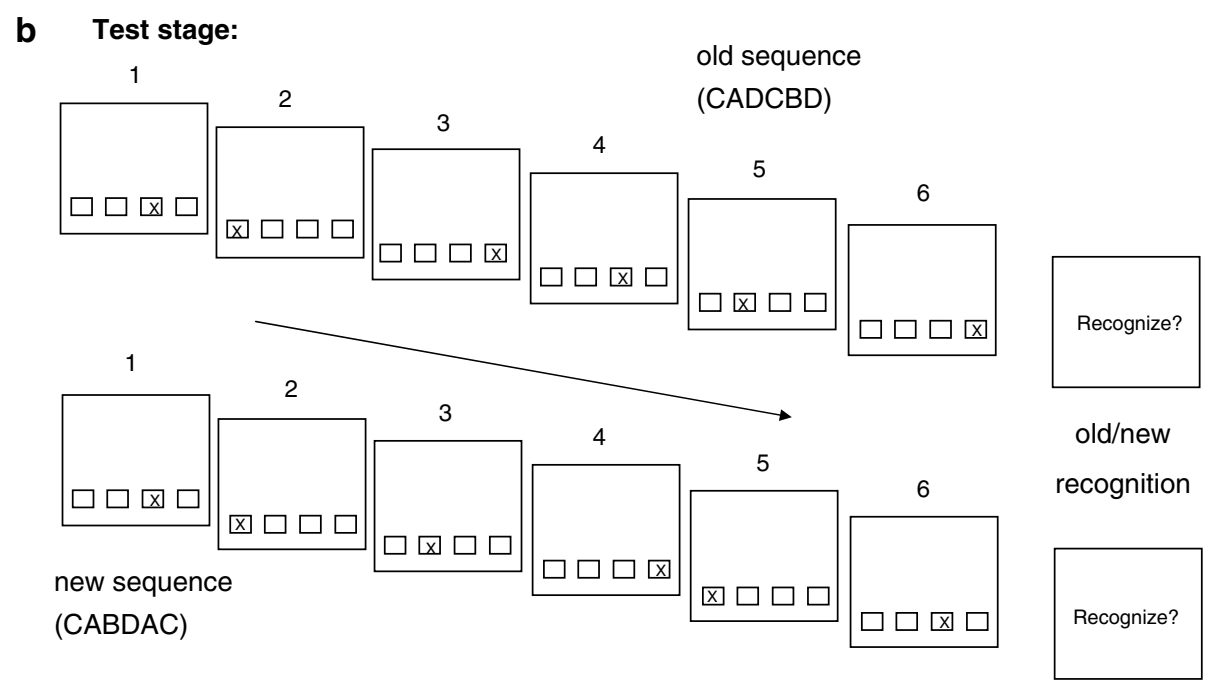

Figure I Schematic illustration of the sequential reaction time (SRT) task used here. (a) During the training stage participants reacted to several blocks (nine in Study I, 12 in Study 2) each comprising 100 target localization trials. Letters A-D refer to the four screen locations from left to right. The target followed a noisy repeating sequence derived from a base sequence such as that shown here. On 85\% of trials the target appeared at the location programmed by the base sequence according to its locations on the preceding two trials (eg DB is followed by A). On 15\% of trials it appeared at an improbable location programmed by a different base sequence (eg DB is followed by C) as illustrated by the bold and underlined targets in the sample sequence. (b) Test stage procedure for measuring contextual priming. Each test trial comprised a sequence of six targets which was either derived from the base sequence (eg CADCBD) or a new sequence (eg CABDAC). Participants reacted to each target within the sequence, as in the training stage, and then rated that sequence as old or new. The first two targets of the test sequence could not be anticipated as the base sequence presented every possible target pairing equally often. However target 3 and subsequent targets could be anticipated by knowledge of the sequence. Hence if the target appeared at locations $C$ and $A$ then its next location was likely to be location $D$. A measure of priming was obtained for each successive target in the test sequence by comparing RTs to the old and new sequences.

The test phase (Figure 1b) involved a performance and recognition test for chunks from the training sequence. Before the test phase, participants were informed that they would now be presented with short sequences of six target movements some of which were part of the training sequence and some of which were not. They were requested to respond to each target as before and then judge whether the sequence was old or new and give a rating of how confident they were in their judgment. Ratings were made on a 6-point scale where $1=$ 'CERTAIN I have NOT SEEN this sequence before,' $2=$ 'FAIRLY CERTAIN I have NOT SEEN this sequence before,' $3=$ 'GUESS I have NOT SEEN this sequence before,' $4=$ 'GUESS I have SEEN this sequence before,' $5=$ 'FAIRLY CERTAIN I have SEEN this sequence before,' and $6=$ 'CERTAIN I have SEEN this sequence before.' The terms 'old' and 'new' should be read as relative as the method of generating probabilistic sequences in the training stage exposes participants to subsequences of both the training and the alternate sequence, but with the latter being much rarer.

There were 24 test sequences in total, presented in a randomized order for each participant. Of the sequences, 12 were constructed by starting at each serial location of SOC1 and 12 were constructed by starting at each serial location of SOC2. Thus, the SOC1 test sequences were old for participants trained on SOC1 and new for those trained on SOC2 and vice versa for the SOC2 sequences. All targets were determined by probable transitions. Two dependent variables were measured in the test stage: RTs to the targets and recognition judgments.

In Study 2, to maximize motivation in the recognition test, participants were instructed that their recognition test performance would be rewarded incrementally depending on performance. In addition to their turn-up fee, for every correct response they received an extra 20 pence.

\section{RESULTS}

Figure 2 shows each participant's mean RT obtained over the training phase, plotted separately for the amnesic and control groups of Study 1 (Figure 2a) and for the three groups of the pharmacological study (Study 2; Figure 2b) and for each type of target (probable and improbable). Probable and improbable targets elicited similar RTs at the outset but with further training probable targets tended increasingly to elicit faster RTs than improbable ones in both groups, indicative of sequence learning. 


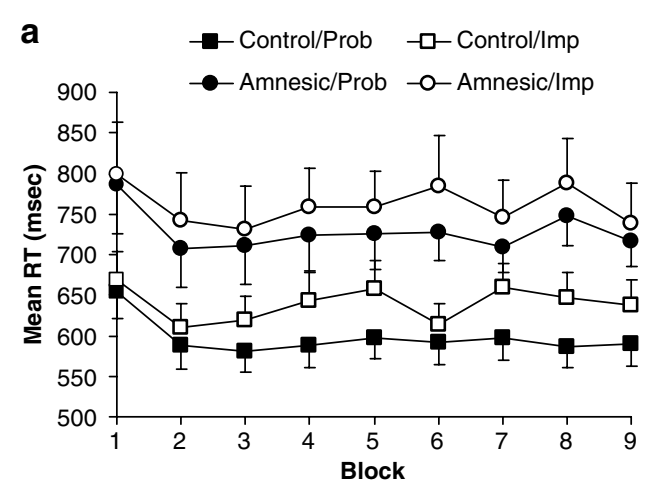

b
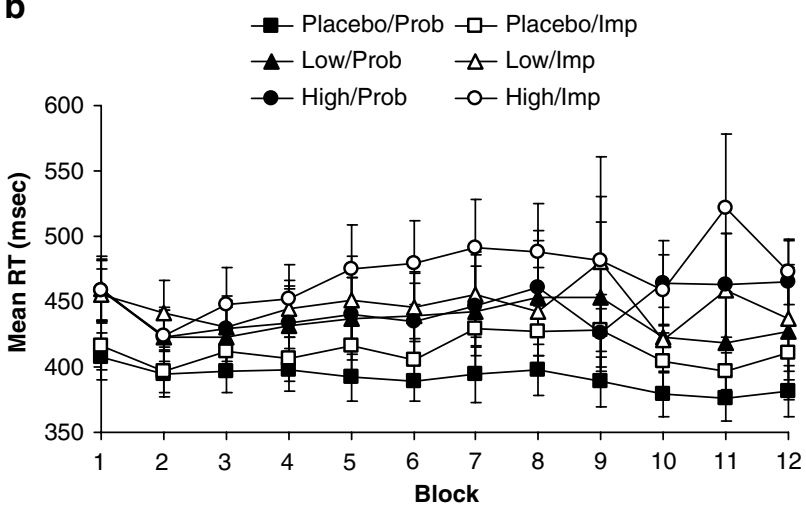

Figure 2 Mean RT (msec) across training blocks. (a) In Study I, a group of amnesic individuals $(n=10)$ and a group of matched controls $(n=22)$ performed nine blocks of 100 trials with the SRT task. Probable targets were consistent with the generating sequence whereas improbable ones were not. Error bars represent SE. Although slower overall than the controls, the amnesic group discriminated as well as the controls between probable and improbable targets, indicative of sequence learning. (b) Corresponding data for Study 2 in which participants $(n=15-16$ per group) were administered $7.5 \mathrm{mg}$ (low dose) or $15 \mathrm{mg}$ (high dose) of diazepam or a placebo. The placebo group was faster than the control group of Study I because the participants were a much younger sample. Probable/improbable discrimination again emerged in all groups.

Consistent with previous results (Schvaneveldt and Gomez, 1998; Shanks et al, 2003), a probable/improbable difference emerged quite early (within 200-300 trials). More importantly, the extent of sequence learning was similar across groups within each study: neither organic nor psychopharmacological amnesia impaired sequence acquisition. This conclusion was statistically confirmed by ANOVAs on mean RTs with probability and block as within-subjects variables and group as a between-subjects variable. This analysis revealed significant main effects of probability in both studies $\left(\mathrm{F}_{1,30}=29.53\right.$ and $\mathrm{F}_{1,44}=40.97$, respectively, $p<0.05$ ) demonstrating learning of the sequences. As shown in Figure 2, learning emerges as early in amnesia as in control conditions. In Study 1 the effects of block $\left(\mathrm{F}_{8,240}=3.41\right)$ and group $\left(\mathrm{F}_{1,30}=6.75\right)$ were also significant $(p<0.05)$, the latter effect reflecting the fact that the amnesic individuals were slower overall. None of the remaining effects or interactions was significant in either study.

To investigate further whether there was any differential effect of amnesia on the magnitude of overall learning during the training phase, a composite measure of learning across blocks was calculated for each study by obtaining a difference score (probable-improbable trials) for each participant, and taking the mean difference score across blocks. In Study 1 the mean difference scores were -42 (control) and -32 (amnesic) $\mathrm{ms}\left(\mathrm{t}_{30}<1, p>0.1\right)$, confirming no difference in learning in the two groups. In the lowdose group of Study 2 differentiation was consistent across blocks 3-12, except blocks 8 and 10 in the low-dose group and block 10 in the high-dose group for which mean RTs for probable trials were numerically slower than mean RTs for improbable trials. A composite measure of learning across blocks was again calculated and the mean difference scores were -21 (placebo), -11 (low dose), and $-25 \mathrm{~ms}$ (high dose), $\mathrm{F}_{2,46}=2.07, p>0.1$, confirming no difference in the magnitude of the learning effect in the three groups. Hence, while both groups learned to respond faster to probable than improbable targets, there was no hint that this learning proceeded at a different rate in the two groups.

Error rates were generally low and varied between 1 and $11 \%$ in Study 1 and between 2 and 13\% in Study 2 .

Figure 3 shows mean RTs for old and new test sequences at each sequence position in each group. On targets 1 and 2, which could not be predicted, there was no evidence of an old/new RT difference but across targets 3-6, which were predictable, RTs were about $30 \mathrm{~ms}$ faster per target in the control participants for old compared to new sequences, indicative of response priming. To clarify the contextual priming data, a priming score was obtained as in earlier studies (Shanks and Perruchet, 2002) by taking average RTs for new test sequences and subtracting the corresponding RTs for old sequences (excluding positions 1 and 2). These scores were then averaged across targets 3 and 4 and across targets 5 and 6. The resulting scores are shown in Figure 4. In Study 1 (Figure 4a), priming was substantial (about $40 \mathrm{~ms}$ per response) in the control group both at targets $3 / 4$ and at targets $5 / 6$, but was entirely absent in the amnesic group, and these priming scores differed significantly $\left(\mathrm{F}_{1,30}=4.44\right.$, $p<0.05$; there was no effect of target and no interaction between target and group, $p>0.05$ in each case). Priming was reliably above-chance in the control group $\left(\mathrm{t}_{21}=3.85\right.$, $p<0.05)$ but not in the amnesic group $(p>0.1)$. Whereas $18 / 22$ control participants showed a priming effect overall, only $5 / 10$ amnesic individuals did; this proportion $(5 / 10)$ is exactly in line with the chance level that would be expected if there is no priming.

Inspection of the data from Study 2 (Figure $4 \mathrm{~b}$ ) reveals that in the placebo group, old sequences were executed faster than new ones at targets $3 / 4$ and 5/6. In contrast, no such priming effect was observed in the high-dose group who closely resemble the amnesic group from Study 1 . In the low-dose group priming was not present at targets $3 / 4$ but emerged at targets 5/6. These observations are confirmed by an ANOVA on RTs with target (3/4 and 5/6) as a within-participant variable and dose as a betweenparticipants variable. The analysis revealed a significant effect of target $\left(\mathrm{F}_{1,44}=3.05, p<0.05\right)$. More importantly, there was a significant interaction between target and dose $\left(F_{2,44}=3.27, p<0.05\right)$, reflecting the pattern of results described above. The main effect of dose was not significant.

One samples $t$-tests against chance (0) showed that for all groups the targets $1 / 2$ score failed to reach significance 

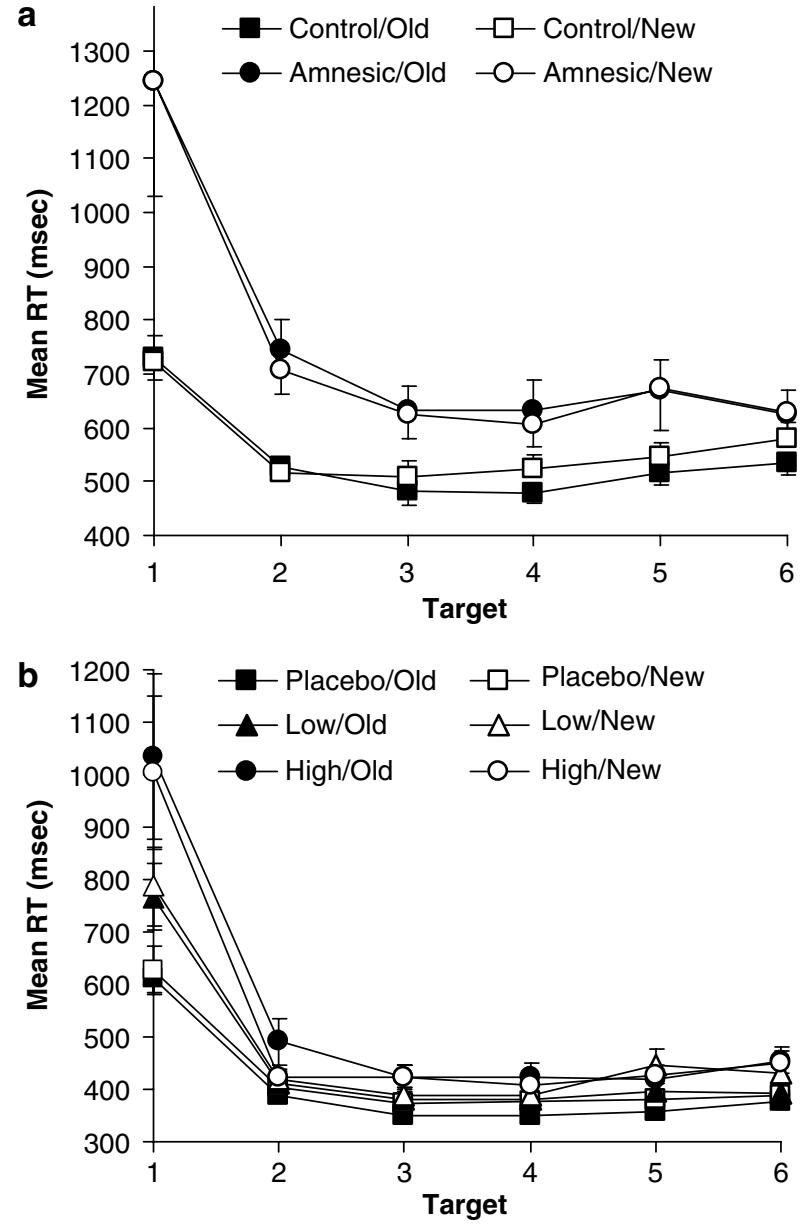

Figure 3 Mean reaction time to targets $\mid-6$ in old and new test sequences. Targets 3-6 were predictable from the preceding targets whereas targets $1-2$ were not predictable. Error bars depict standard errors. Responses were particularly slow at location I as this is the first trial of each sequence and includes response preparation time. Priming is evident as faster responding to targets in old compared to new sequences. (a) In Study I priming is evident at target 3, the earliest possible point, and continues across the remaining targets. Hence two elements of context are sufficient to induce response priming. No priming was observed in the amnesic group, however. (b) Corresponding data for Study 2. Derived priming measures are shown in Figure 4.

( $p>0.05$ in each case), as expected. The targets $3 / 4$ and $5 / 6$ difference scores were significantly above 0 in the placebo group $\left(\mathrm{t}_{15}=4.24\right.$ and 2.18 , respectively, $p$ 's $\left.<0.05\right)$ but not in the high-dose group $\left(\mathrm{t}_{15}=-0.40\right.$ and 0.09 , respectively, $p$ 's $>0.1)$. In the low-dose group, priming was absent at targets $3 / 4\left(\mathrm{t}_{14}=1.22, p>0.05\right)$ but significant at targets $5 / 6$ $\left(\mathrm{t}_{14}=2.57, p<0.05\right)$, and the increase in priming in this group between targets $3 / 4$ and $5 / 6$ was also statistically reliable $\left(\mathrm{t}_{14}=2.36, p<0.05\right)$. Clearly the disruption of contextual priming caused by diazepam was both dose dependent and graded in the sense that it depended on the number of preceding context locations. While 13/16 participants in each of the control and low-dose groups showed a priming effect overall, only $7 / 15$ individuals in the high-dose group did, this proportion being in line with chance.

Mean recognition ratings for old and new sequences are shown in Figure 5 (higher ratings correspond to greater
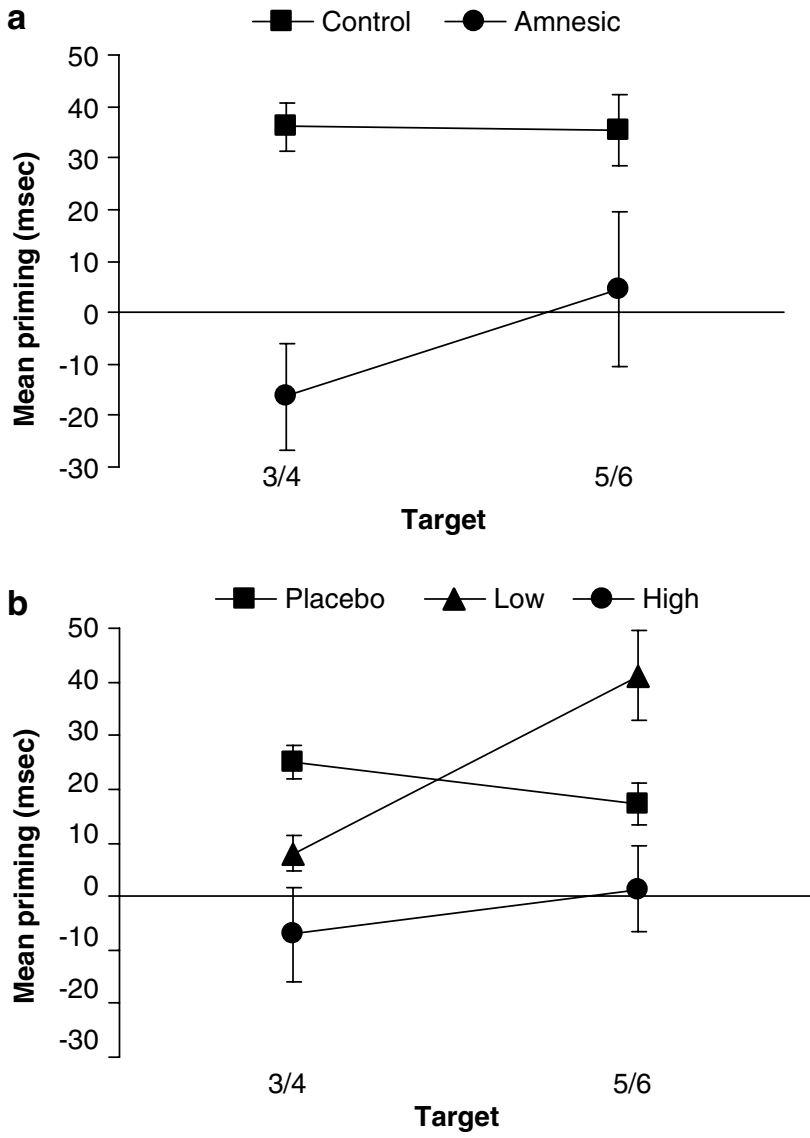

Figure 4 Mean priming scores derived from the data in Figure 3. RTs at each location of the old sequences are subtracted from the corresponding RTs from new sequences and the results combined across targets $3 / 4$ and 5/6 for clarification. Error bars depict standard errors. (a) In Study I priming is evident at all locations in the control group but no priming is observed in the amnesic group. (b) In Study 2 priming occurs at all locations in the placebo group, is absent across all locations in the high-dose group, and develops for targets $5 / 6$ in the low-dose group $(p<0.05$ for dose by location interaction).

old $\square$ new

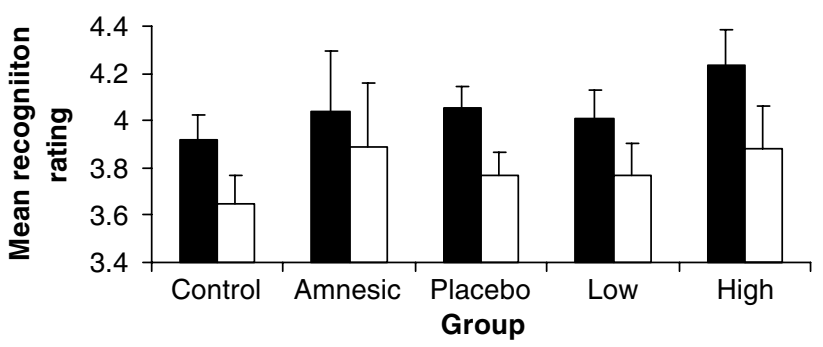

Figure 5 Mean recognition ratings ( $\mid=$ certain new, $6=$ certain old $)$ to old and new test sequences in each group. Error bars depict SE.

confidence that the sequence is old). It is clear that participants in the control group of Study $1 \quad\left(\mathrm{t}_{21}=2.54\right.$, $p<0.05$ ) (Figure 5a) and in all three groups of Study 2 $\left(\mathrm{t}_{15}=3.41, \mathrm{t}_{14}=1.99\right.$, and $\mathrm{t}_{15}=2.14$, for the placebo and low- and high-dose groups, respectively, $p<0.05$ in each case) (Figure 5b) were able to differentiate between old and new sequences. However, discrimination was at chance in 
the amnesic group in Study $1(\mathrm{t}<1, p>0.1)$ confirming that the task meets one of the key criteria of implicit learning, that individuals with medial temporal lobe damage can show learning without recognition. When the data were analyzed by signal detection theory, identical conclusions were reached: discrimination $\left(\mathrm{d}^{\prime}\right)$ was significant in all groups except the organic amnesics.

\section{DISCUSSION}

These results reveal that sequence learning per se is not impaired in temporal lobe or diazepam-induced amnesia, but that the expression of sequence knowledge is. To access and express sequence knowledge, normal participants require only two elements of context in our procedure in order to show priming on the next and subsequent responses. In contrast, organic amnesic participants failed to show such priming at all. Indeed, even the 6th response, with five previous elements of context, did not show priming. Under diazepam, a dose-related pattern of context priming was observed, with no priming at all under a high dose (15 mg) but priming from four (but not two) previous locations obtained under a low dose $(7.5 \mathrm{mg})$.

We interpret the test results in terms of a reduced efficiency in amnesia to extract and express contextual support. Amnesia reduces the degree to which contingencies are learned between successive (targets $n-2$ and $n-1$ predicting target $n$ ) and longer-range targets (eg targets $n-4$ and $n-3$ predicting target $n$ ). However, these contingencies are learned to some extent such that when enough context is provided, the priming associated with each contingency combines to yield a detectable influence on response latencies, in much the same way that a child may be able to recite the entire alphabet but may not be able to give the successor to a particular letter. For controls, learning of these contingencies is much more efficient and even the shortest-range contingency (targets $n-2$ and $n-1$ predicting target $n$ ) is strong enough to yield priming. Moreover, we assume that, with a probable/improbable ratio of $0.85 / 0.15$, this short-range contingency is learned in controls to an asymptotic level by the end of training so that the longer-range contingencies provide no increments to priming. Put differently, control participants learn small 'chunks' better than amnesics. While both adults and children can recite the whole alphabet, adults tend also to know smaller chunks (eg $\mathrm{Q} \rightarrow \mathrm{R}$ ) than children.

Our results demonstrate increasing demands on contextual support when memory is disrupted but do not allow us to say how much context is needed to induce priming in severe amnesia (ie organic or high drug dose). We know that it is greater than five elements as that amount was insufficient, and we know that it is less (probably much less) than 100 elements as priming was no lower overall in the amnesic groups than in the control groups across the training blocks. It might be thought that we could analyze the early trials in each block of the training stage to see where priming starts to be detectable, but this is impossible as there are insufficient improbable targets at each position in the sequence against which to compare responding to the probable targets. Future studies with longer sequences in the test stage should be able to quantify exactly how much context is required to induce priming.
Our findings are consistent with a fundamental role of medial temporal lobe structures in the use of context to retrieve information. Our performance measure was an indirect or procedural one, and not a declarative measure. That is to say, we measured learning and contextual influence in a task in which participants were instructed simply to respond as fast as possible to targets. Despite this, amnesia induced either pharmacologically or by brain lesion caused disruption of performance. We conclude that the critical medial temporal lobe structures related to amnesia serve the function of binding information together, regardless of whether that information is retrieved explicitly (declaratively) or implicitly (procedurally). Hence, as with other illustrations of impaired contextual learning in amnesia (Chun and Phelps, 1999; Park et al, 2004; Ryan et al, 2000), our findings do not support the idea that the hippocampus and medial temporal lobes control declarative memory alone.

The fact that sequence learning was unaffected by amnesia in the training stage, combined with significant memory impairment on other measures, is consistent with the view that the SRT task measures implicit or procedural learning. Both our organic- and diazepam-induced amnesics were impaired on standard tests of declarative memory (eg prose recall), confirming that medial temporal lobe memory systems were affected. Previous research has also observed normal sequence learning during the training stage in both organic and pharmacologically induced amnesia. Several studies have documented normal sequence learning in organic amnesia on the SRT task (Reber and Squire, 1994, 1998) (but see Curran, 1997). Some previous research has looked at sequence learning under differing doses of amnestic drugs (Bishop et al, 1996) and some has assessed single doses (Knopman, 1991; Martin et al, 2002; Nissen et al, 1987). These studies have found that neither scopolamine, nor the BZDs lorazepam and oxazepam, affect procedural learning under these circumstances. Hence, consistent with the procedural/declarative view, medial temporal lobe memory structures are not essential for procedural sequence learning. They plainly are essential, however, for context-primed expression of that knowledge.

It might be speculated that because participants in these studies had to make recognition decisions in the test stage, the entire test (including the priming measure) was transformed into a test of declarative memory. On this view, the experimental groups who demonstrated impaired priming did so because they were attempting to use declarative memory to recall the sequence and because their relevant declarative memory was inadequate to allow this. This view predicts that priming and declarative memory (including recognition) should be correlated: the better the sequence is recalled, the more efficiently the target's position can be anticipated and the faster the sequence can be executed. However, priming and recognition clearly were not correlated: the organic amnesic and high-dose diazepam groups were equally impaired in terms of priming but differed in their recognition performance, while the low- and high-dose diazepam groups did not differ in recognition but did in terms of priming. Although the relationship between priming and recognition is a complex one that is still not well understood (Conroy et al, 2005; Kinder and Shanks, 2003; Shanks et al, 2003), and the 
difference in recognition performance between the organic and high-dose amnesics deserves further study, it cannot be argued that priming in the present studies was based on declarative memory.

Recently, Manns and Squire (2001) have suggested a modification of the declarative/procedural framework, which is consistent with our and other related (Chun and Phelps, 1999; Ryan et al, 2000) findings. Manns and Squire reported some evidence that learning in the contextual cuing task of Chun and Phelps (1999) (another task which measures implicit contextual priming) is impaired in individuals with extensive medial temporal lobe amnesia (including the hippocampus) but not in ones with more restricted hippocampal damage. On the basis of this they suggested that the function of the hippocampus is indeed restricted to declarative learning and that nondeclarative contextual or relational learning depends on other medial temporal lobe structures. Unfortunately, this conjecture is difficult to test. First, it is very rare for individuals to have lesions confined to the hippocampus alone. Secondly, the fact that such individuals appear to show normal implicit contextual priming may simply be due to the fact that their amnesia is more mild than that in individuals with extensive medial temporal lobe damage (this was plainly the case in Manns' and Squire's participants).

Brain imaging studies may play a useful role here. To the extent that they are able to distinguish between hippocampal activity on the one hand and that in entorhinal, perirhinal, and parahippocampal cortices on the other, they may shed light on the involvement of these different structures in contextual learning and priming as well as in declarative learning. Many studies have measured regional brain activation during the SRT task (eg Honda et al, 1998; Willingham et al, 2002) but these have invariably examined performance across a long block of learning trials instead of directly manipulating contextual support as in the studies reported here. Such tests are inadequate for examining contextual priming. Plainly, brain imaging analyses during the test phase of our procedure, comparing activity with different amounts of context, would offer considerable promise of resolving the precise role of the hippocampus and nearby structures in learning and performance.

\section{ACKNOWLEDGEMENTS}

We thank Denise Waller and Louise Healey for their assistance with Study 1. This research was supported by a grant from the United Kingdom Economic and Social Research Council.

\section{REFERENCES}

Bishop DVM (1989). Test for the Reception of Grammar. Manual: Oxford, UK.

Bishop KI, Curran HV, Lader M (1996). Do scopolamine and lorazepam have dissociable effects on human memory systems? A dose-response study with normal volunteers. Exp Clin Psychopharmacol 4: 292-299.

Bond AJ, Lader MH (1974). The use of analogue scales in rating subjective feelings. Br J Med Psychol 47: 211-218.
Chun MM, Phelps EA (1999). Memory deficits for implicit contextual information in amnesic subjects with hippocampal damage. Nat Neurosci 2: 844-847.

Cleeremans A, Destrebecqz A, Boyer M (1998). Implicit learning: news from the front. Trends Cogn Sci 2: 406-416.

Cleeremans A, Jiménez L (1998). Implicit sequence learning: the truth is in the details. In: Stadler MA, Frensch PA (eds). Handbook of Implicit Learning. Sage: Thousand Oaks, CA. pp 323-364.

Cleeremans A, McClelland JL (1991). Learning the structure of event sequences. Journal of Experimental Psychology: General 120: 235-253.

Cohen NJ, Eichenbaum H (1993). Memory, Amnesia, and the Hippocampal System. MIT Press: Cambridge, MA.

Cohen NJ, Ryan J, Hunt C, Romine L, Wszalek T, Nash C (1999). Hippocampal system and declarative (relational) memory: summarizing the data from functional neuroimaging studies. Hippocampus 9: 83-98.

Conroy MA, Hopkins RO, Squire LR (2005). On the contribution of perceptual fluency and priming to recognition memory. Cogn Affect Behav Neurosci 5: 14-20.

Coughlan AK, Hollows SE (1985). The Adult Memory and Information Processing Battery: Test Manual. St James' University Hospital: Leeds, UK.

Curran HV (2000). Psychopharmacological approaches to human memory. In: Gazzaniga MS (ed). The New Cognitive Neurosciences. MIT Press: Cambridge, MA. pp 797-804.

Curran T (1997). Higher-order associative learning in amnesia: evidence from the serial reaction time task. J Cogn Neurosci 9: 522-533.

Destrebecqz A, Cleeremans A (2001). Can sequence learning be implicit? New evidence with the process dissociation procedure. Psychonom Bull Rev 8: 343-350.

Elman JL (1990). Finding structure in time. Cogn Sci 14: 179-212.

Gabrieli JDE (1998). Cognitive neuroscience of human memory. Ann Rev Psychol 49: 87-115.

Grant AD, Berg EA (1948). A behavioral analysis of reinforcement and ease of shifting to new responses in a Weigl-type card sorting. J Exp Psychol 38: 404-411.

Honda M, Deiber M-P, Ibáñez V, Pascual-Leone A, Zhuang P, Hallett M (1998). Dynamic cortical involvement in implicit and explicit motor sequence learning: a PET study. Brain 121: 2159-2173.

Kinder A, Shanks DR (2003). Neuropsychological dissociations between priming and recognition: a single-system connectionist account. Psychol Rev 110: 728-744.

Knopman D (1991). Unaware learning versus preserved learning in pharmacologic amnesia: similarities and differences. $J$ Exp Psychol: Learning Memory Cogn 17: 1017-1029.

Knowlton BJ (2005). Cognitive neuropsychology of learning and memory. In: Lamberts K, Goldstone RL (eds). Handbook of Cognition. Sage: London. pp 365-381.

Manns JR, Squire LR (2001). Perceptual learning, awareness, and the hippocampus. Hippocampus 11: 776-782.

Martin J, Matthews A, Martin F, Kirkby KC, Alexander J, Daniels B (2002). Effects of lorazepam and oxazepam on perceptual and procedural memory functions. Psychopharmacology 164: 262-267.

Nelson H (1991). National Adult Reading Test: Second Edition. Manual. NFER-Nelson: Windsor.

Nissen MJ, Knopman DS, Schacter DL (1987). Neurochemical dissociation of memory systems. Neurology 37: 789-794.

O’Reilly RC, Rudy JW (2001). Conjunctive representations in learning and memory: principles of cortical and hippocampal function. Psychol Rev 108: 311-345. 
Park H, Quinlan J, Thornton E, Reder LM (2004). The effect of midazolam on visual search: implications for understanding amnesia. Proc Natl Acad Sci 101: 17879-17883.

Reber PJ, Squire LR (1994). Parallel brain systems for learning with and without awareness. Learning Memory 1: 217-229.

Reber PJ, Squire LR (1998). Encapsulation of implicit and explicit memory in sequence learning. J Cogn Neurosci 10: 248-263.

Reed J, Johnson P (1994). Assessing implicit learning with indirect tests: determining what is learned about sequence structure. J Exp Psychol: Learning Memory Cogn 20: 585-594.

Rey A (1941). L'examen psychologique dans les cas d'encéphalopathie traumatique (The psychological examination in cases of traumatic brain damage). Arch Psychol 28: 286-340.

Rudy JW, Sutherland RJ (1995). Configural association theory and the hippocampal formation: an appraisal and reconfiguration. Hippocampus 5: 375-389.

Ryan JD, Althoff RR, Whitlow S, Cohen NJ (2000). Amnesia is a deficit in relational memory. Psychol Sci 11: 454-461.

Schvaneveldt RW, Gomez RL (1998). Attention and probabilistic sequence learning. Psychol Res 61: 175-190.

Shallice T, Burgess PW (1991). Deficits in strategy application following frontal lobe damage in man. Brain 114: 727-741.

Shanks DR (2005). Implicit learning. In: Goldstone RL (ed). Handbook of Cognition. Sage: London. pp 202-220.

Shanks DR, Perruchet P (2002). Dissociation between priming and recognition in the expression of sequential knowledge. Psychonom Bull Rev 9: 362-367.
Shanks DR, St John MF (1994). Characteristics of dissociable human learning systems. Behav Brain Sci 17: 367-447.

Shanks DR, Wilkinson L, Channon S (2003). Relationship between priming and recognition in deterministic and probabilistic sequence learning. J Exp Psychol: Learning Memory Cogn 29: 248-261.

Squire LR (1994). Declarative and nondeclarative memory: multiple brain systems supporting learning and memory. In: Schacter DL, Tulving E (eds). Memory Systems 1994. MIT Press: Cambridge, MA. pp 203-231.

Thurstone LL, Thurstone TG (1962). Primary Mental Abilities (Rev.). Science Research Associates: Chicago.

Warrington EK (1984). The Recognition Memory Test. NFERNelson: Windsor.

Warrington EK, James M (1991). The Visual Object and Space Perception Battery. Thames Valley Test Company: Bury St Edmunds, UK.

Wechsler D (1981). Wechsler Adult Intelligence Scale: Revised. Manual. The Psychological Corporation: New York.

Willingham DB, Nissen MJ, Bullemer P (1989). On the development of procedural knowledge. J Exp Psychol: Learning Memory Cogn 15: 1047-1060.

Willingham DB, Salidis J, Gabrieli JDE (2002). Direct comparison of neural systems mediating conscious and unconscious skill learning. J Neurophysiol 88: 1451-1460.

Wilson B, Cockburn J, Baddeley A (1985). The Rivermead Behavioural Memory Test. Thames Valley Test Company: Reading, UK. 